\title{
Effect of external fixation rod coupling in computed tomography
}

\author{
Carlos A. Peña-Solórzano ${ }^{1}$ (1) Matthew R. Dimmock ${ }^{1} \cdot$ David W. Albrecht $^{2} \cdot$ David M. Paganin $^{3} \cdot$ Richard B. Bassed $^{4,5}$. \\ Mitzi Klein ${ }^{6}$. Peter C. Harris ${ }^{7,8}$
}

Received: 19 February 2018 / Accepted: 11 September 2018 / Published online: 15 September 2018

(c) The Author(s) 2018

\begin{abstract}
External fixation is a common tool in the treatment of complex fractures, correction of limb deformity, and salvage arthrodesis. These devices typically incorporate radio-opaque metal rods/struts connected at varying distances and orientations between rings. Whilst the predominant imaging modality is plain film radiology, computed tomography (CT) may be performed in order for the surgeon to make a more confident clinical decision (e.g. timing of frame removal, assessment of degree of arthrodesis). We used a fractured sheep leg to systematically assess CT imaging performance with a Discovery CT750 HD CT scanner (GE Healthcare) to show how rod coupling in both traditional Ilizarov and hexapod frames distorts images. We also investigated the role of dual-energy CT (DECT) and metal artefact reduction software (MARS) on the visualisation of the fractured leg. Whilst mechanical reasons predominantly dictate the rod/strut configurations when building a circular frame, rod coupling in CT can be minimised. Firstly, ideally, all or all but one rod can be removed during imaging resulting in no rod coupling. If this is not possible, strategies for configuring the rods to minimise the effect of the rod coupling on the region of interest are demonstrated, e.g., in the case of a four-rod construct, switching the two anterior rods to a more central single one will achieve this goal without particularly jeopardising mechanical strength for a short period. It is also shown that the addition of DECT and MARS results in a reduction of artefacts, but also affects tissue and bone differentiation.
\end{abstract}

Keywords Computed tomography $\cdot$ Rod coupling $\cdot$ Dual-energy CT $\cdot$ Metal artefact reduction $\cdot$ Metal artefacts

The work was performed at Western Health, Footscray Hospital, Gordon St, Footscray, Melbourne, VIC 3011, Australia.

Carlos A. Peña-Solórzano

carlos.penasolorzano@monash.edu

Matthew R. Dimmock

matthew.dimmock@monash.edu

David W. Albrecht

david.albrecht@monash.edu

David M. Paganin

david.paganin@monash.edu

Richard B. Bassed

richard.bassed@vifm.org

Mitzi Klein

Mitzi.Klein@monash.edu

Peter C. Harris

chris.harris@rch.org.au

1 Department of Medical Imaging and Radiation Sciences, Monash University, Wellington Rd, Clayton, Melbourne, VIC 3800, Australia
2 Clayton School of Information Technology, Monash University, Wellington Rd, Clayton, Melbourne, VIC 3800, Australia

3 School of Physics and Astronomy, Monash University, Wellington Rd, Clayton, Melbourne, VIC 3800, Australia

4 Victorian Institute of Forensic Medicine, 57-83 Kavanagh St., Southbank, Melbourne, VIC 3006, Australia

5 Department of Forensic Medicine, Monash University, Wellington Rd, Clayton, Melbourne, VIC 3800, Australia

6 Australian Synchrotron, 800 Blackburn Rd, Clayton, Melbourne, VIC 3168, Australia

7 The Royal Children's Hospital Melbourne, 50 Flemington Road, Parkville, Melbourne, VIC 3052, Australia

8 Department of Orthopaedic Surgery, Western Health, Footscray Hospital, Gordon St, Footscray, Melbourne, VIC 3011, Australia 


\section{Introduction}

Circular external fixation is one of several tools available for the orthopaedic surgeon to use in the management of a number of orthopaedic conditions [1], including the treatment of complex fractures, correction of limb deformity, and salvage arthrodesis.

During the post-operative period, imaging is performed to assess, amongst other things, alignment and progress of healing (callus formation, union, etc.). Whilst the predominant imaging modality is plain film radiology, computed tomography (CT) may be performed when plain film radiology does not give sufficient detail of a region of interest (ROI) in order for the surgeon to make a more confident clinical decision (e.g. timing of frame removal, assessment of degree of arthrodesis).

The frame components, being relatively radio-opaque, have the capacity to either obscure the view of the region of interest (predominantly in the case of plain film radiology) or distort the quality of the image to some degree (in the case of CT). For both of these imaging modalities, strategies to minimise this fall under three general categories. Firstly, the surgeon has some degree of choice as to the positioning of certain components (e.g. rings and rods) relative to the region of interest. Secondly, the way in which the patient is positioned during image acquisition can be optimised [2]. Thirdly, some components, such as the connecting rods, can be temporarily removed or repositioned during imaging. With CT there is an additional strategy; many modern scanners have the facility to reduce the effect of metal artefacts through the use of dual-energy CT (DECT) and/or metal artefact reduction algorithms [3-6]. However, such scanner settings are not specific to the individual patient/frame construct, and so not only do they have varying ability to reduce metallic artefacts, but in addition they may also adversely affect image quality in general.

Since there are situations in which the orthopaedic surgeon may rely heavily on the quality of the CT image, this study focuses on the nature of the adverse effect of metal rods/struts on the quality of the image and on how to optimise the image quality in the presence of metallic components.

\section{Materials and methods}

The leg of a sheep that had been euthanised for reasons unrelated to this research was used. A mid-shaft oblique fracture was created by making a single drill hole followed by the use of an osteotome whilst bending the bone. The soft tissues were left in place.

The frame consisted of two 130-mm aluminium rings (Taylor Spatial Frame, Smith \& Nephew), spaced 196 mm apart. Two construct types were studied. Construct one represents the traditional Ilizarov frame, where the rings are connected by threaded stainless steel rods (Ilizarov, Smith $\&$ Nephew) (see Fig. 1b). Construct two is a hexapod, where

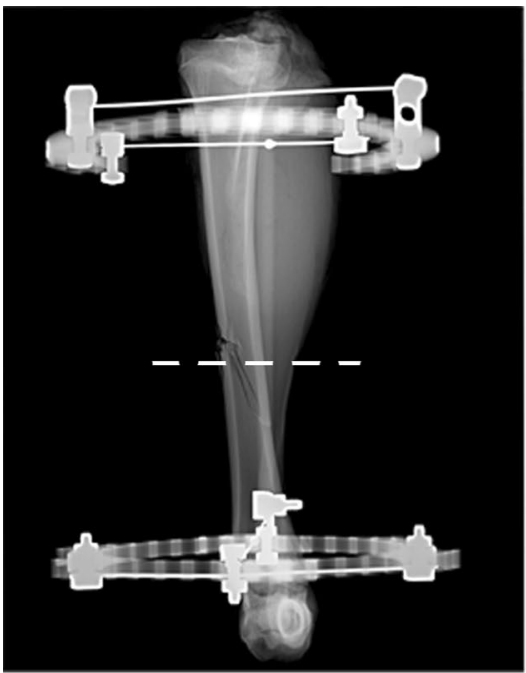

$\mathbf{a}$

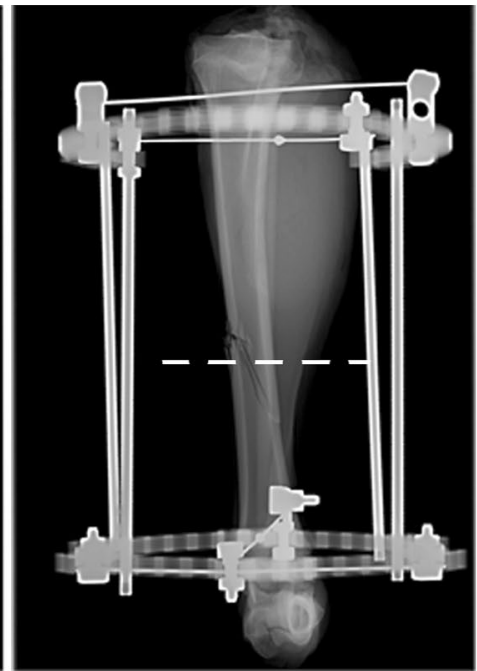

$\mathbf{b}$

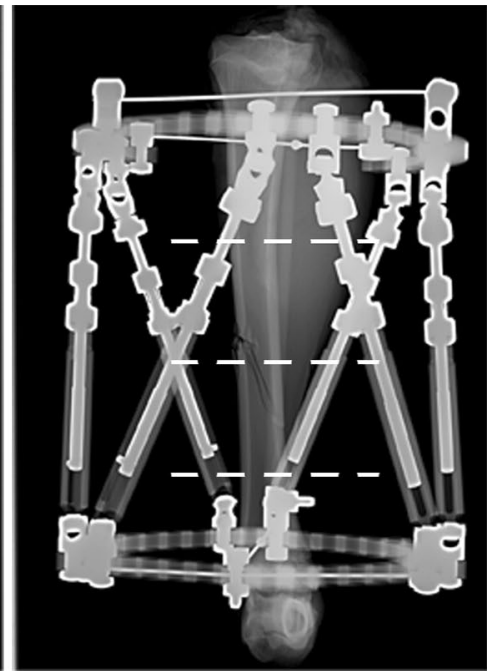

$\mathbf{C}$

Fig. 1 X-rays (anterior-posterior views) showing various frame constructs. a Initial positioning of the rings. b Ilizarov frame (4 rods). c Taylor Spatial Frame. Dotted lines show the position of the slices used for the study 
the rings are connected by six struts attached at the outer mounting holes of the tabs (Taylor Spatial Frame, Smith $\&$ Nephew) (see Fig. 1c). For the Ilizarov frame, the rods were varied both in number and in configuration, whilst for the Taylor Spatial Frame (TSF), only one configuration was used, with all 6 struts at the same length. Each ring was attached to the bone using two crossed and tensioned wires. The rings were connected with three plastic threaded rods, which allowed a baseline scan to be performed without any metal between the rings whilst ensuring that the fracture position remained unchanged. The frame was taped to the $\mathrm{CT}$ bed in an orthogonal orientation, ensuring that a constant and idealised position was maintained for all study images. To further ensure standardisation of slice acquisition, the proximal ring was used as a reference level for each axial slice (Fig. 2).

The scans were performed with a Discovery CT750 HD CT scanner (GE Healthcare), which has the facility for both DECT and metal artefact reduction software (MARS). The frame/leg construct was scanned initially at $100 \mathrm{kVp}$, which represents the single-energy setting that would typically be used in the presence of metal. It was then scanned at dual energy $(80 / 140 \mathrm{kVp})$ with and without MARS. A single axial slice is composed of $512 \times 512(262,144)$ pixels, whilst the field of view chosen for this study resulted in one pixel being equivalent to $0.39 \mathrm{~mm} \times 0.39 \mathrm{~mm}$ of scanned area. Each individual pixel is a measure of relative radiodensity and is given a value termed a Hounsfield unit (HU). Water (at standard pressure and temperature) has a HU of zero,

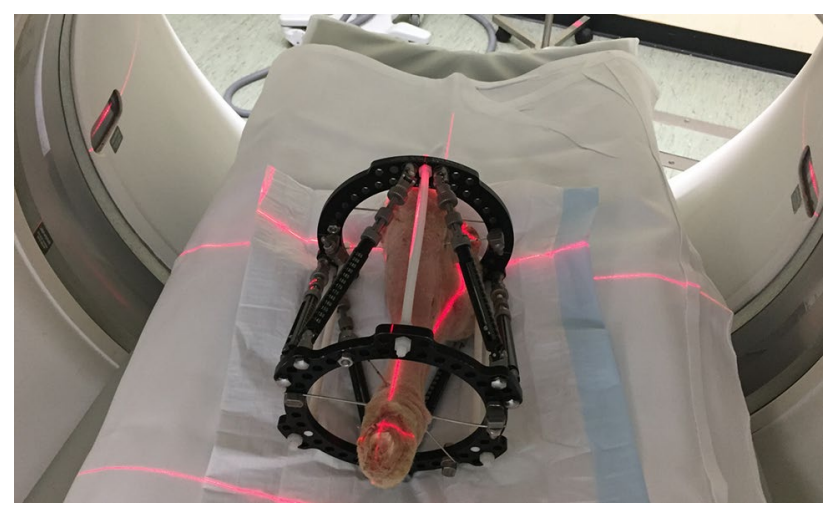

Fig. 2 Photograph of the frame/leg construct in the CT scanner whilst anything of lesser radiodensity is a negative value and anything of greater radiodensity is a positive value [7].

For the purposes of this study, the pixel values are displayed in two distinct ways: a traditional axial 2D image and a histogram. For the 2D image, the Hounsfield units are displayed as a greyscale, with negative values being increasingly dark and positive values being increasingly white (Fig. 3a, left). We used display settings with a centre of $300 \mathrm{HU}$ and a window width of $2800 \mathrm{HU}$, which is not a typically used window width, but allows us to better observe the metal artefacts affecting the background of the images. The histogram is a graphical representation of the number of pixels of particular HU that are present in the field of view (Fig. 3a, right). To make the histogram more specific, a ROI around the tibia was defined (Fig. 3b, left); this removes the peak produced by air, making it easier to appreciate any change in the other two peaks (Fig. 3b, right). Whilst the histogram gives no spatial appreciation of individual pixels, it allows a quantitative analysis of the effect of the metal artefact on image quality by using Gaussian fitting of the bone and soft tissue peaks (Fig. 3b; dashed line; note that a Gaussian function appears as an inverted parabola on a logarithmic scale). The parameters of the peak that were analysed for the Gaussian functions were the mean $\mathrm{HU}$ value and the width (2.35 times the standard deviation).

\section{Results}

Results are displayed as 2D images and ROI histogram, using the scale shown in Fig. 3. The ROI varies according to the slice being analysed, with the shape of an approximately elliptical shape that follows the outer boundary of the bone. Unless otherwise mentioned, the images show scans performed with single-energy CT.

\section{Baseline image}

Figure $4 \mathrm{a}$ shows the 2D image and ROI histogram of an axial slice through the fracture site in a construct where there is no metal between the rings (although the plastic rods are evident). It represents the best quality image that can be obtained and therefore serves as the baseline for comparison with all subsequent images, where the presence of varying degrees of metal produces some degree of image degradation. 
$\mathbf{a}$
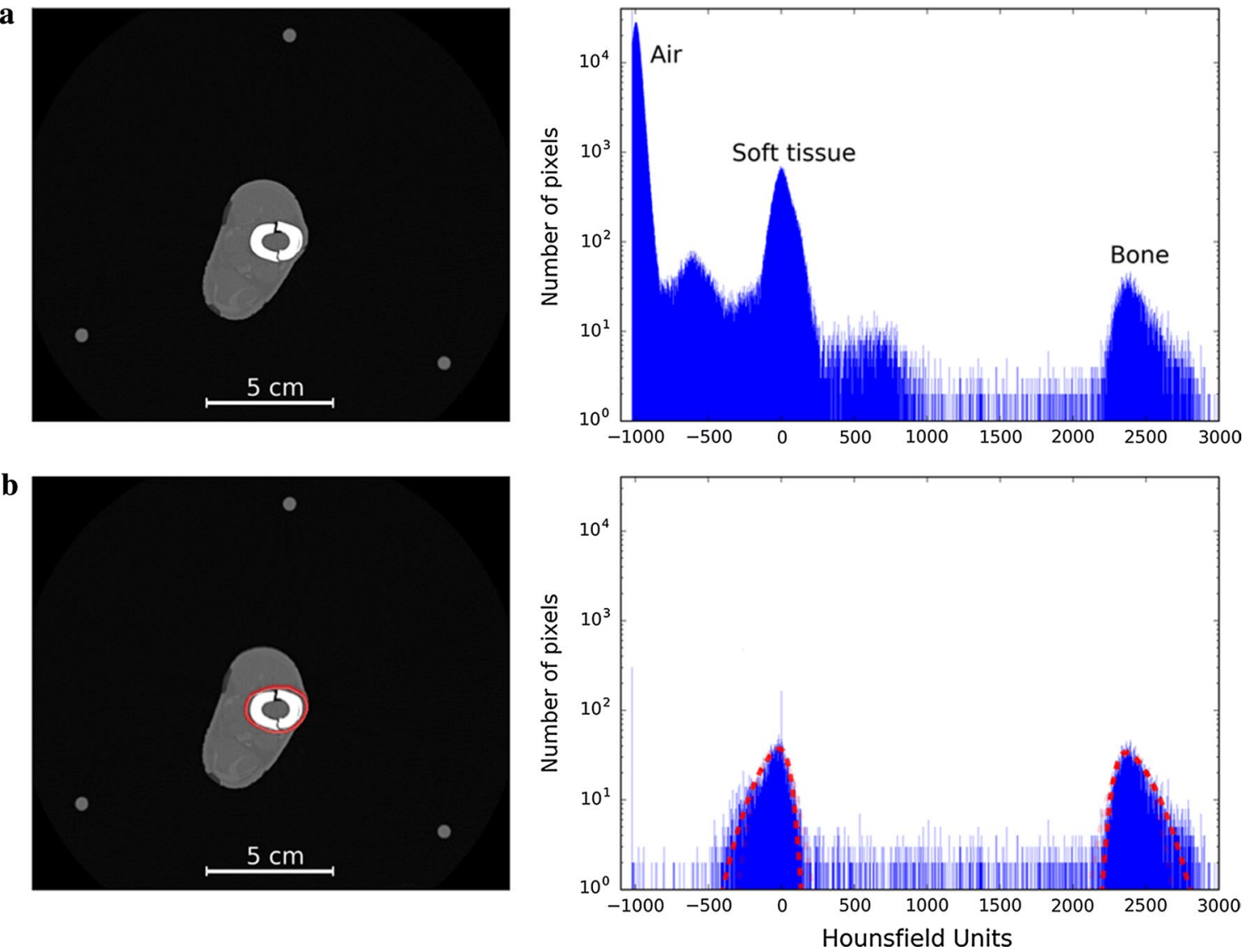

Fig. 3 Example of information used for the analyses. a 2D axial slice (left) and histogram of whole image (right), with the three peaks representing air (black), soft tissue (grey), and bone (white). b 2D axial slice showing the region of interest circled in red (left) and histogram of the pixels inside the chosen area (right), where mean HU value and width are determined for bone and soft tissue using Gaussian fitting, shown in dotted lines

\section{Ilizarov frame}

The presence of a single metal rod (Fig. 4b) causes fine streaks artefacts to radiate from it, but they have little effect on the quality of the image. On the histogram, the bone and soft tissue peaks are similar to those obtained from the image without rods.

When two rods are used, the effect on the region of interest is highly dependent upon where they are placed (Fig. 5). On the 2D image it can be seen that the rods act as a couple, producing a broader and more noticeable streak that runs between them. This streak has a dark centre and bright edges. Where this streak crosses a part of the image that is already dark (Fig. 5a), its effect is negligible. However, if it crosses the region of interest, then its effect on detail is more pronounced (Fig. 5b). This can be appreciated on the histogram as a change in the spikes (wider bases and a change in the mean values).

Interestingly, when 3 rods are used, the effect on the region of interest is minimal (Fig. 6a); broad dark streaks with bright edges still occur between rods, but because they do not cross the ROI, they have little adverse effect. For the four-rod construct there are six such streaks, but only two of them cross the region of interest (Fig. 6b). The histogram shows a change in the spikes (wider bases), indicating loss of contrast and therefore image quality. 
$\mathbf{a}$
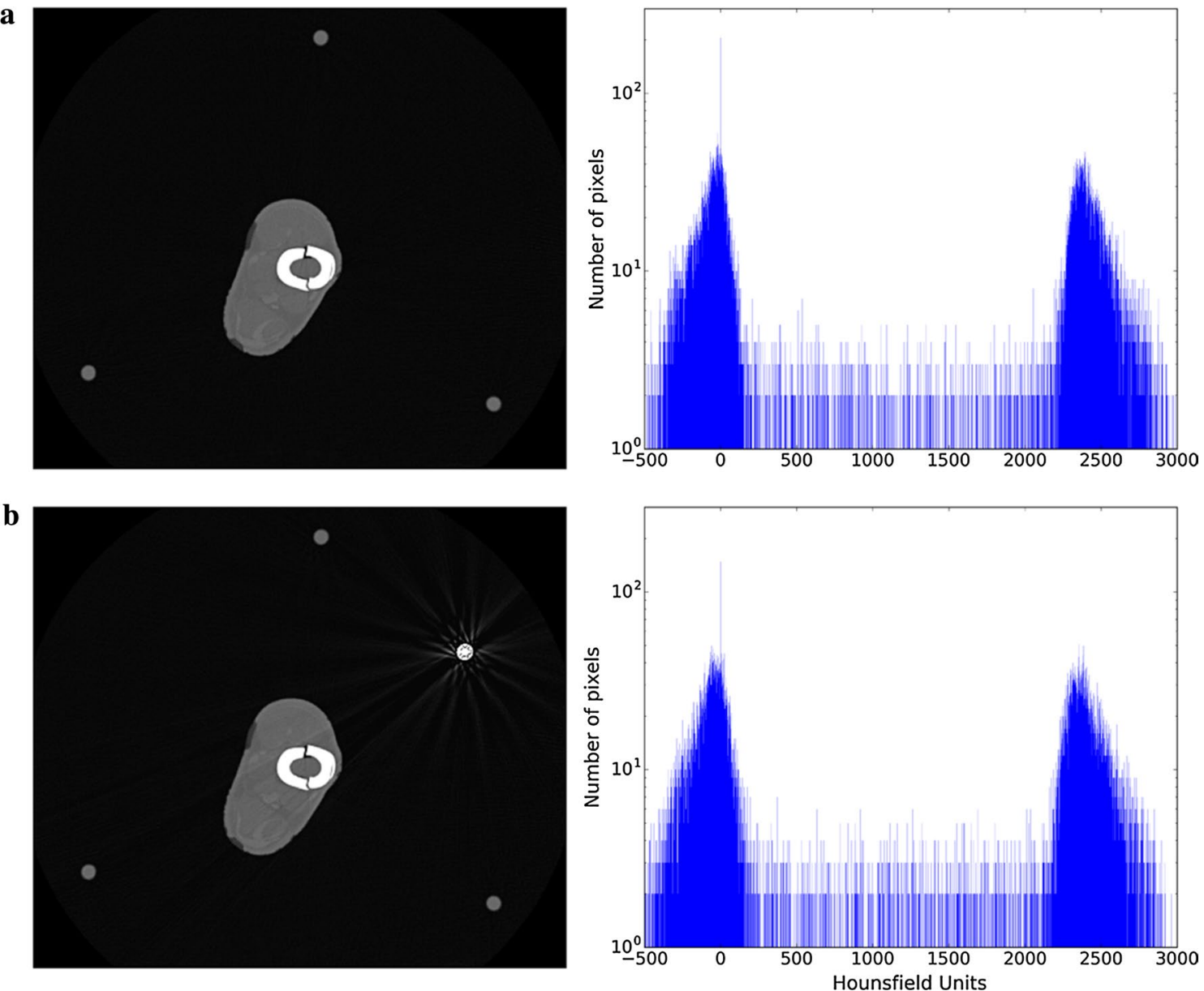

Fig. 4 a Axial slice and histogram of the fracture site when there are not metallic components present. b Axial slice and histogram of the fracture site when only one rod is present

\section{Taylor Spatial Frame}

Geometric shape: The oblique orientation of the struts in the frame results in a distinctive geometric pattern of broad streaks, the nature of which differs according to the level scanned (Fig. 7). There are slices where the ROI is not directly affected by the streak (Fig. 7a), whilst in others, it is crossed several times (Fig. 7b).

Strut material: The makeup of the strut differs along its length, with one end being tubular casing (Fig. 7c) and the other end being a solid rod (Fig. 7a). In the middle, these two materials overlap to some degree (Fig. 7b). The severity of the broad streaks depends on which part of the strut is present in the particular slice.

\section{Dual-energy scanning/metal artefact reduction software}

Figures 8 and 9 show the effect of techniques used to reduce metal artefacts with the four-rod Ilizarov and TSF constructs, 

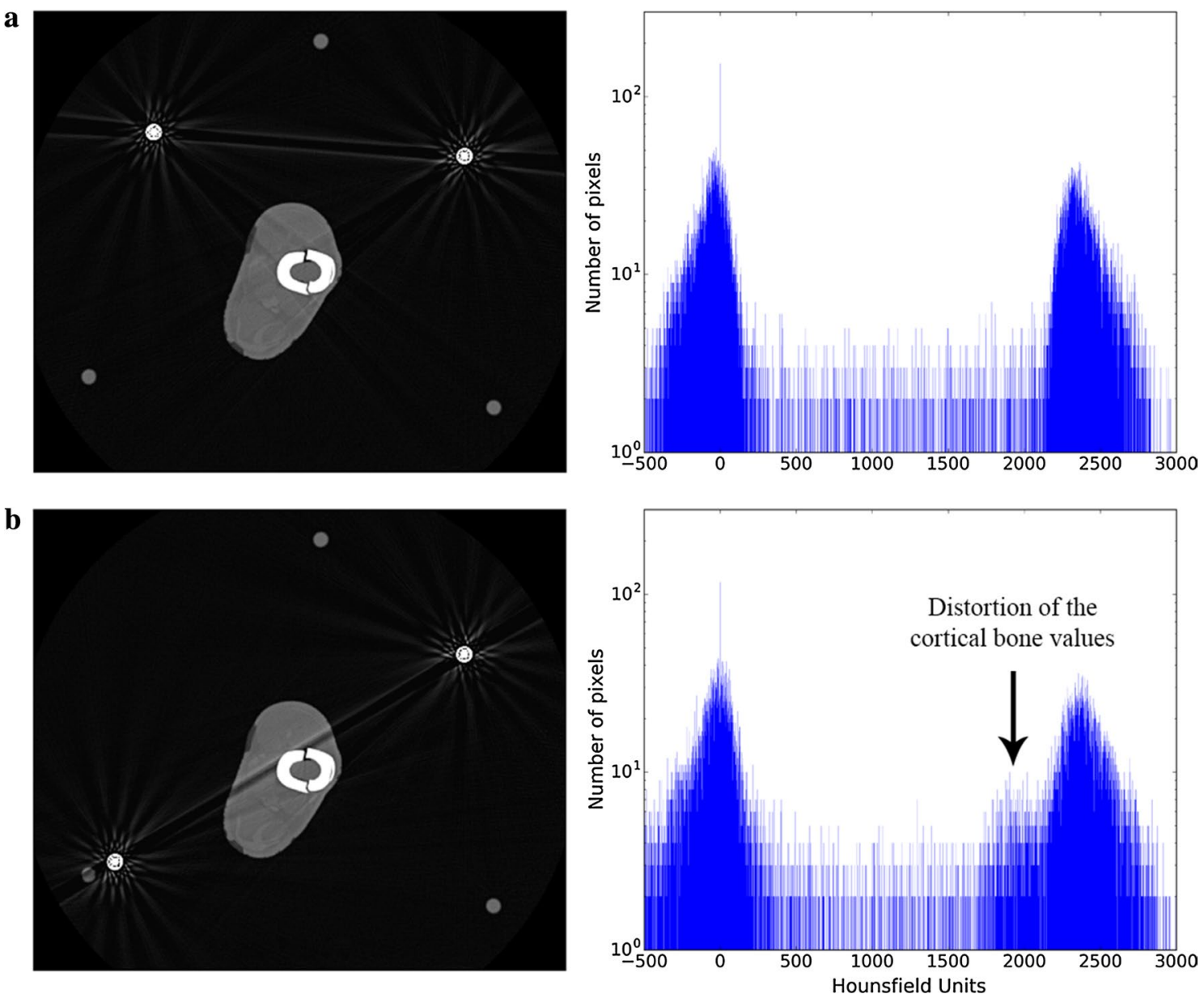

Fig. 5 Axial slice and histogram of the fracture site when there are two metal rods. a The rods are located at the same side of the leg. $\mathbf{b}$ The rods are located at opposite sides of the leg. The rod coupling goes through the ROI

respectively. Figures $8 \mathrm{a}$ and $9 \mathrm{a}$ present the single-energy scans shown previously, whilst Figs. $8 \mathrm{~b}$ and $9 \mathrm{~b}$ are scans performed using DECT, and Figs. 8c and 9c present scans using DECT and MARS. Both of these modalities are associated with a decrease in the overall clarity of the image, particularly so with the MARS. For the histograms, the higher-energy scan has altered the $\mathrm{HU}$ value for bone from its mean of 2400 on the single-energy scan to around 1100 , whilst the addition of MARS further results in the peaks tending to merge into one.

\section{Discussion}

Obtaining good-quality images is a key part of the decisionmaking process for patients with circular external fixation. Frequently, nearby metal can obscure or have an adverse effect on details of the images obtained with plain film radiography and CT $[3,8]$.

The idea for this study came about because it is the practice of the senior author $(\mathrm{PCH})$ to remove as many rods as possible (or exchange struts for rods) when CT imaging. Typically, this is done whilst the patient is lying on the CT bed, so that the temporarily weakened frame is not subjected to undue forces. The rods are then reinserted after the scan has been performed and before the patient gets off the bed. Since the scan itself is relatively quick, the surgeon may end up being present during the whole process of removal, scanning, and reattachment. It requires that the scan is performed at a time when the surgeon is available and is clearly time-consuming, for both the surgeon and the CT department. It therefore raises the question of how much benefit is derived by such a practice; is the improvement in image 

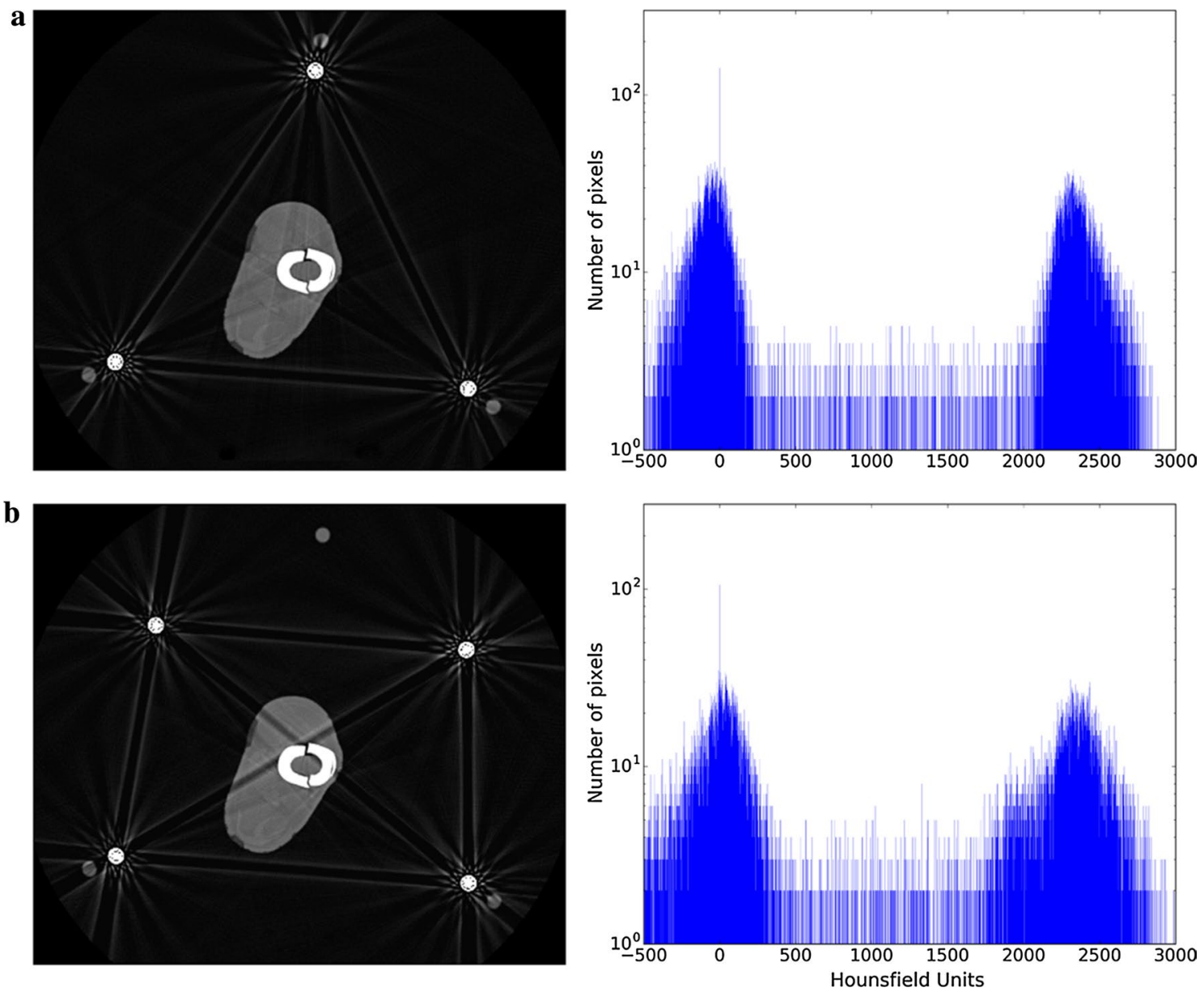

Fig. 6 a Axial slice and histogram of the fracture site when there are three metal rods present. b Axial slice and histogram of the fracture site in the presence of four metal rods

quality really worth all that effort? The aim of our study was therefore to investigate how the presence of rods/struts affects image quality. We chose a sheep leg over a human subject in order to scan it as many times as needed without concern for the adverse effects that ionising radiation can have on living humans.

The degradation of the CT image arises from the interaction between the poly-energetic X-ray beam and dense structures [4], creating two distinctive effects. The first effect (generalised noise proportional in degree to the overall amount of metal present on the axial slice) is seen as fine dark and bright streaks on the image $[9,10]$. In this case, the artefact is distributed fairly proportionately throughout the image. The second effect, which is due to a pairing between rods and struts, causes a more noticeable broad dark streak with surrounding bright edges, and is a function of the helical manner in which the scan is acquired (Fig. 10). Whilst the general existence of artefacts from geometric considerations has been previously acknowledged $[8,11]$, our study focuses on its relevance for circular external fixation, for which we have coined the term rod coupling. Our study demonstrates that, in the case of circular external fixation, it is rod coupling that is the main factor causing degradation of image quality. The histograms show how changes in the values of bone and soft tissue due to the metal artefacts affect the contrast of the image. The spreading of the peaks in the histograms, which corresponds to bone and soft tissue, in general causes a loss of visibility of the trabecular structure which lays between the peaks, and therefore loses definition. 
$\mathbf{a}$

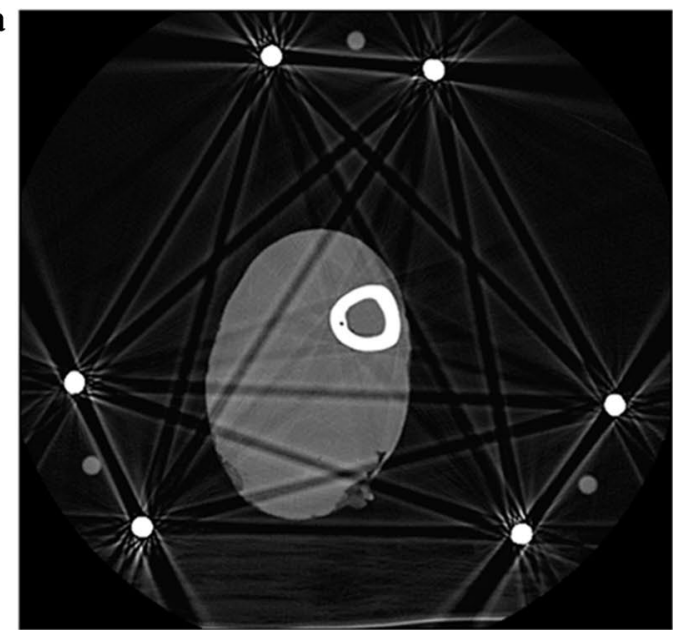

b

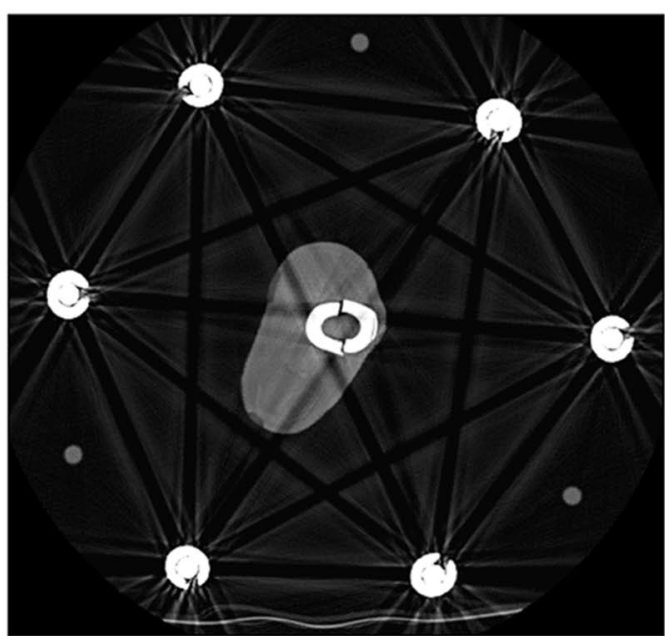

c

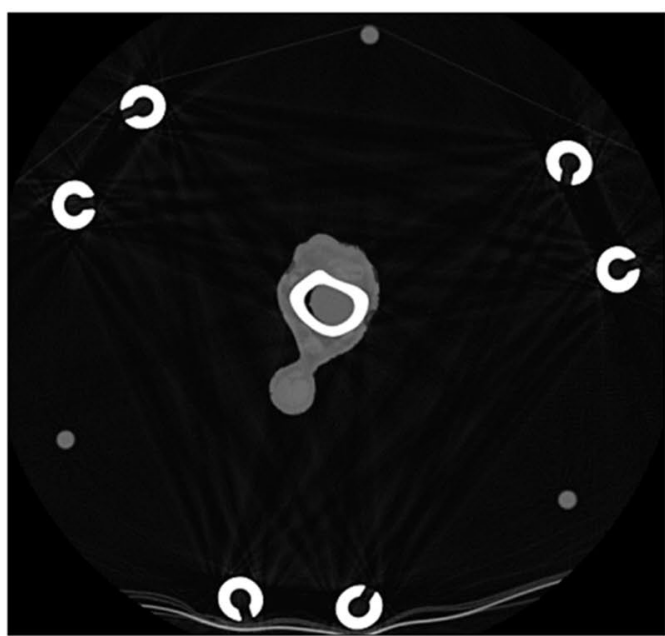

Fig. 7 Axial slices and histograms of the leg when using the TSF. a Slice proximal to fracture site, with only the solid rods of the strut, can be observed. b Slice of the fracture site, showing a mixture of
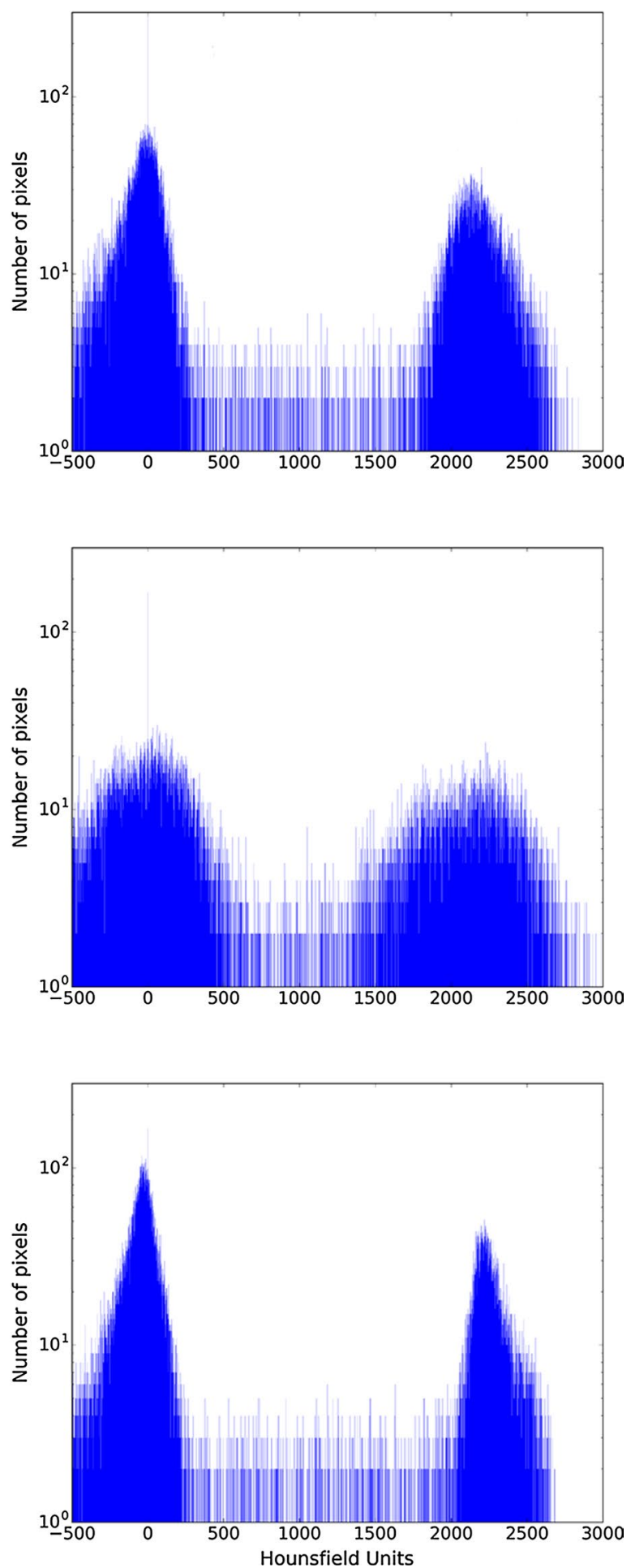

the solid rod and the tubular casing. $\mathbf{c}$ Slice distal to the fracture site, where only the tubular casing of the struts is observed 

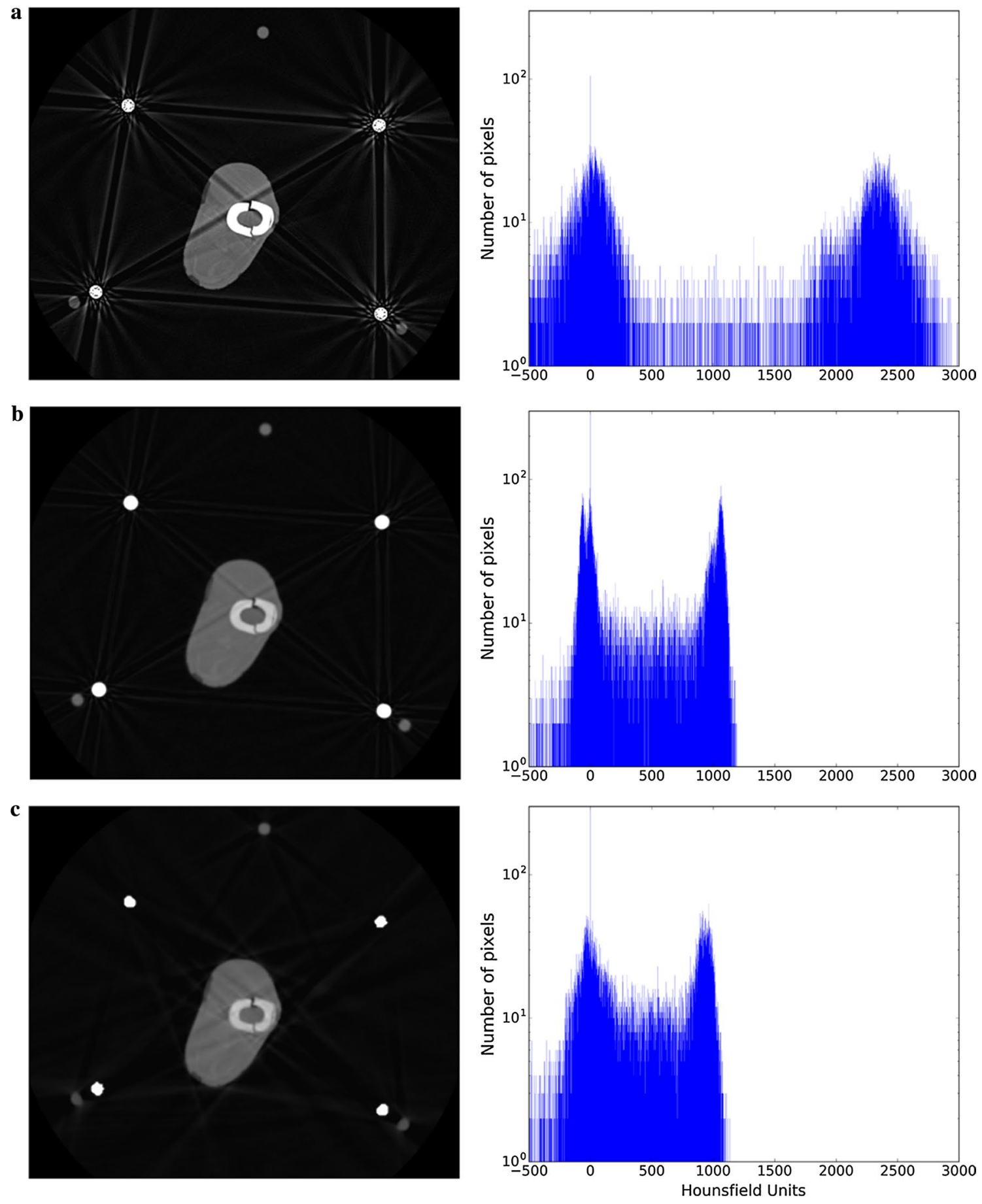

Fig. 8 Effect of metal artefact reduction techniques in image clarity in the presence of 4 metal rods. a Single-energy scan. b DECT. c DECT + MARS. The bone peak moves to the left for DECT and

DECT + MARS, which causes this tissue to become darker in the images when the window is conserved

Whilst we acknowledge that mechanical reasons predominantly dictate the rod/strut configurations when building a circular frame, the effect of rod coupling during CT imaging can be minimised. Firstly, all or all but one rod can be removed during imaging; we see this as the ideal (Fig. 4),

as in these cases there is no rod coupling. If this is not a possibility at that particular time, the second option is to configure the rods in a way that minimises the effect of the rod coupling on the area of interest. It can be appreciated in Fig. 6 that in the case of a four-rod construct, simply 
$\mathbf{a}$
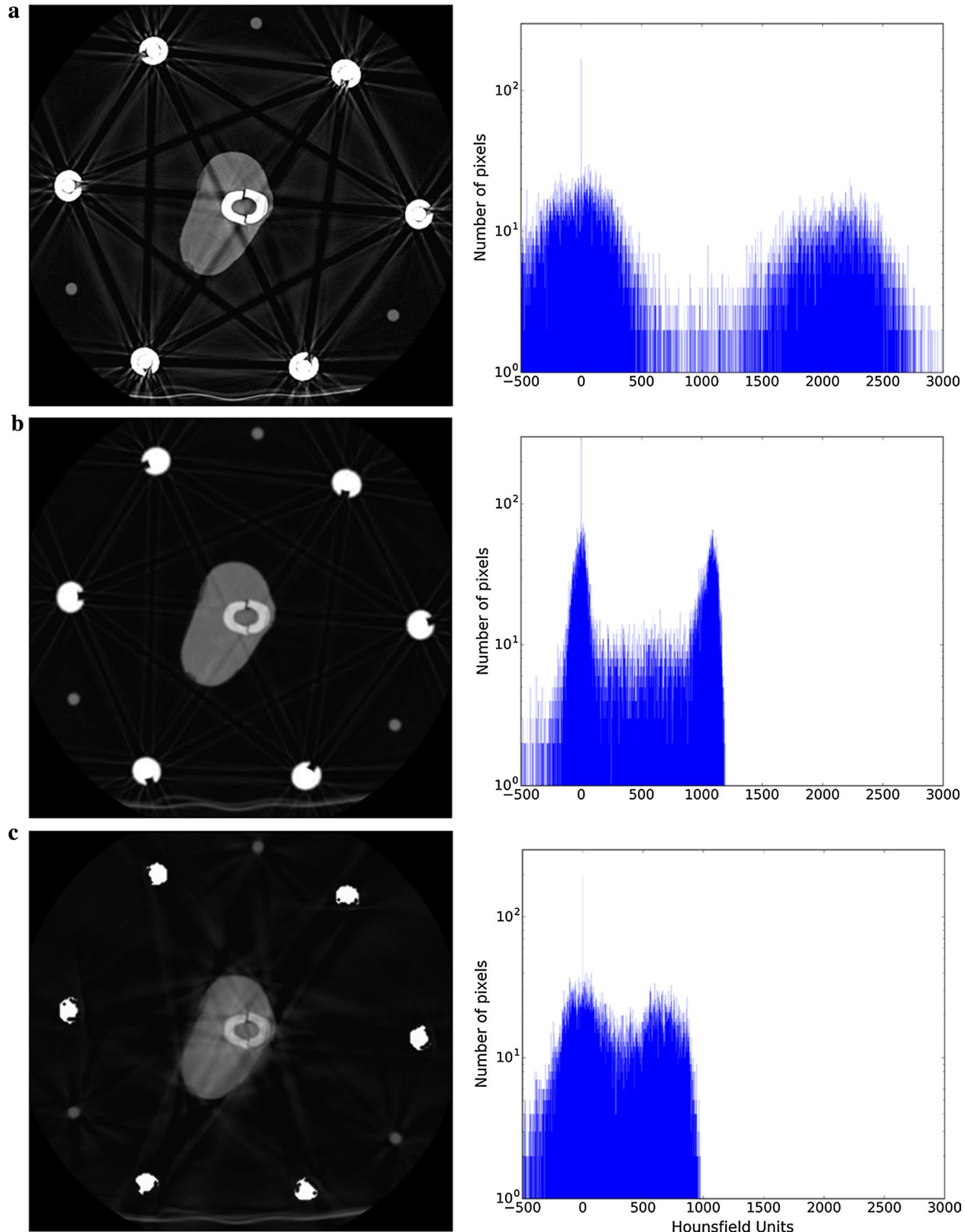

Fig. 9 Effect of metal artefact reduction techniques in image clarity in the presence of the TSF. a Single-energy scan. b DECT. $\mathbf{c}$ DECT + MARS

switching the two anterior rods to a more central single one will achieve this goal without particularly jeopardising mechanical strength for a short period. In the case of hexapod frames, whilst temporarily switching struts for rods would also appear to be a good idea, this can be more timeconsuming, as often with hexapods the rings are not parallel, and therefore requiring the use of dished (conical) washers or the construction of hinges. 
Fig. 10 Helical nature of the CT scanning process, with $\mathrm{X}$-ray source and detectors shown in three positions A-C. When the source completes a full rotation, a reconstruction algorithm converts the information to a 2D image slice. These slices can be displayed individually or assembled together to generate a $3 \mathrm{D}$ volume of the patient
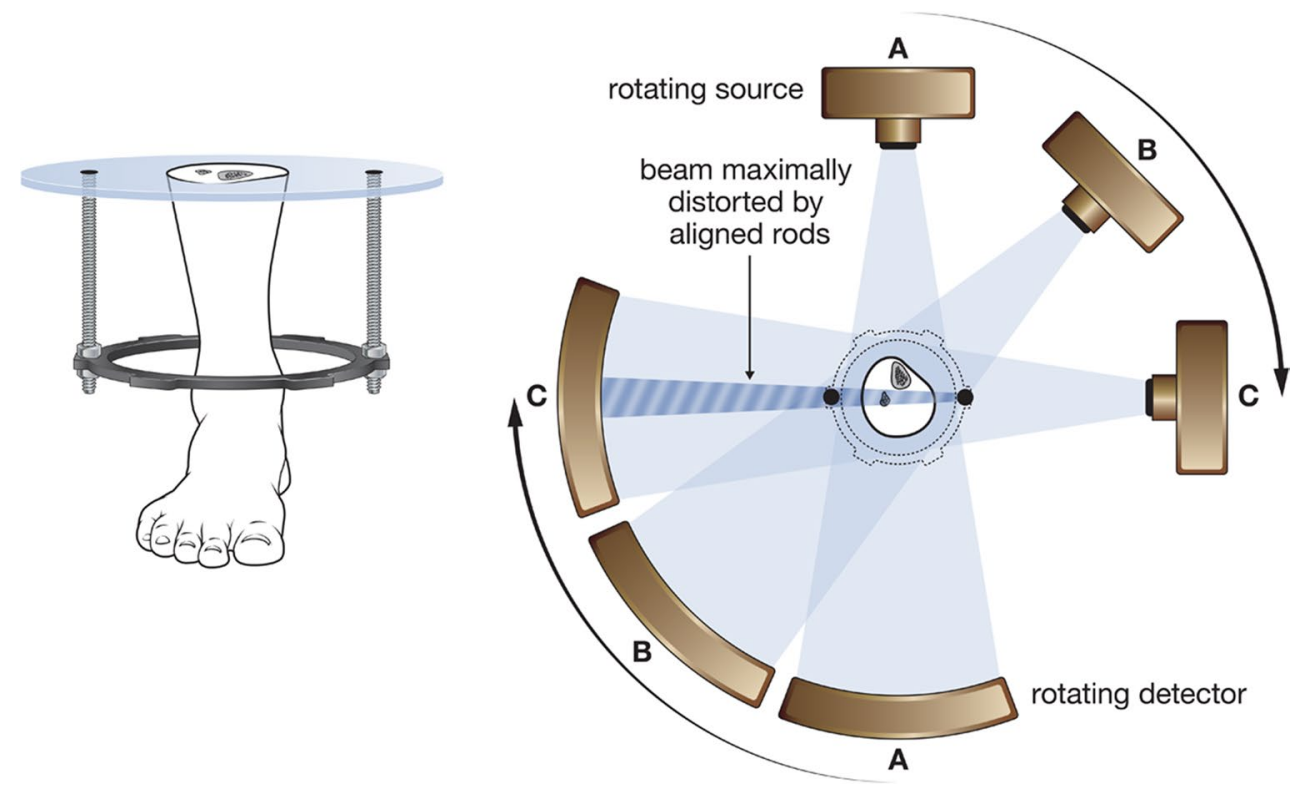

Table 1 Mean and width values for soft tissue and bone peaks as calculated from the histograms when the number of metal rods is changed in single-energy scans

\begin{tabular}{|c|c|c|c|c|}
\hline \multirow[t]{2}{*}{ Number of rods/figure number } & \multicolumn{2}{|l|}{ Soft tissue } & \multicolumn{2}{|l|}{ Bone } \\
\hline & Mean (HU) & Width (HU) & Mean (HU) & Width (HU) \\
\hline 0/Figure $4 a$ & -38 & 207 & 2396 & 251 \\
\hline 1/Figure $4 \mathrm{~b}$ & -48 & 237 & 2377 & 280 \\
\hline 2 (coupling outside ROI)/Fig. 5a & -44 & 244 & 2355 & 298 \\
\hline 2 (coupling crossing ROI)/Fig. 5b & -23 & 284 & 2382 & 388 \\
\hline 3/Figure $6 a$ & -50 & 284 & 2337 & 322 \\
\hline 4/Figure $6 b$ & 16 & 367 & 2344 & 505 \\
\hline
\end{tabular}

Table 2 Mean HU and width values for soft tissue and bone peaks as calculated from the histograms of different slices of the TSF singleenergy CT scan

\begin{tabular}{llllll}
\hline TSF & \multicolumn{2}{l}{$\begin{array}{l}\text { Soft tissue } \\
\text { Figure number }\end{array}$} & & & \multicolumn{2}{l}{ Bone } \\
\cline { 2 - 3 } \cline { 5 - 6 } \cline { 5 - 6 } & Mean (HU) & Width (HU) & & Mean (HU) & Width (HU) \\
\hline Figure 7a & -18 & 256 & & 2160 & 416 \\
Figure 7b & -13 & 682 & & 2111 & 846 \\
Figure 7c & -33 & 190 & & 2231 & 212 \\
\hline
\end{tabular}

Can the CT technician configure the scan in a way that negates the need to remove or reconfigure rods/struts? In DECT, scans are acquired using two different energy settings and then synthesising pseudo-monochromatic scans at a variety of energies $[12,13]$. The advantage of pseudomonochromatic reconstructions is that the effect of streaking due to beam hardening, typically seen in CT imaging, is greatly reduced. Whilst the higher-energy setting reduces photon starvation and beam hardening, it results in some loss of detail (analogous to an over-penetrated plain $\mathrm{X}$-ray). MARS works by evening out unexpected variation in pixels, but our study suggests that the image it produces compares poorly with the original; the mean HU values change considerably.

One limitation of our study relates to assessment of the quality of an image. Image quality is determined by, amongst other things, the resolution and the contrast. In this paper, our assessment/comparison of the quality of the 2D images is purely subjective. Whilst efforts are being made to produce an objective scoring system for the quality of digital images in general [14-16], to our knowledge no such score currently exists for radiography. By making our results section predominantly a display of images, we have allowed the reader to draw their own conclusions about the effects of the various constructs and metal artefact reduction modalities on image quality. Whilst Gaussian-fitting analysis of the histograms produces some degree of quantitative data analysis, it is a relatively crude measure of contrast and is not useful in clinical orthopaedic practice. For readers that are familiar with the attributes of Gaussian peak fitting, the quantitative parameters extracted from the peak-fitting procedure are displayed in Tables 1, 2, 3, and 4 . The general observation is that as the peaks get 
Table 3 Mean HU and width values for soft tissue and bone peaks as calculated from the histograms of four-rod Ilizarov construct scans with single-energy CT, DECT, and DECT + MARS

\begin{tabular}{lcllll}
\hline Ilizarov (4 rods) & \multicolumn{2}{l}{ Soft tissue } & & & Bone \\
\cline { 2 - 3 } Figure number/modality & Mean (HU) & Width (HU) & & Mean (HU) & Width (HU) \\
\hline Figure 8a/single energy & 16 & 367 & & 2344 & 505 \\
Figure 8b/DECT & -22 & 132 & & 1040 & 141 \\
Figure 8c/DECT + MARS & 5 & 284 & & 922 & 181 \\
\hline
\end{tabular}

Table 4 Mean HU and width values for soft tissue and bone peaks as calculated from the histograms of TSF construct scans with single-energy CT, DECT, and DECT + MARS

\begin{tabular}{|c|c|c|c|c|}
\hline \multirow{2}{*}{$\begin{array}{l}\text { TSF } \\
\text { Figure number/modality }\end{array}$} & \multicolumn{2}{|l|}{ Soft tissue } & \multicolumn{2}{|l|}{ Bone } \\
\hline & Mean (HU) & Width (HU) & Mean (HU) & Width (HU) \\
\hline Figure $9 \mathrm{a} /$ single energy & -13 & 682 & 2111 & 846 \\
\hline Figure 9b/DECT & -4 & 125 & 1067 & 160 \\
\hline Figure 9c/DECT + MARS & 13 & 430 & 618 & 486 \\
\hline
\end{tabular}

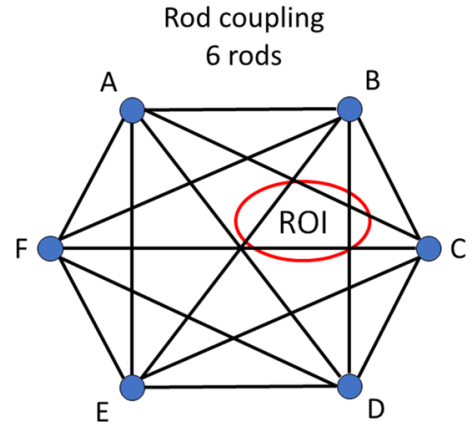

$\mathbf{a}$

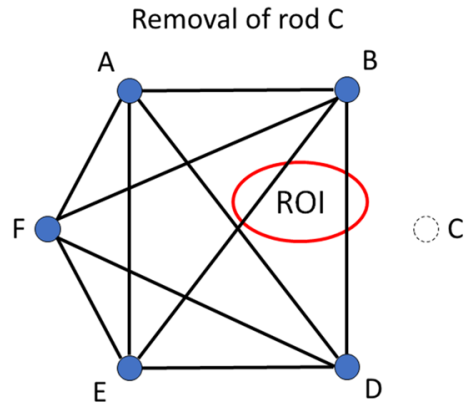

b

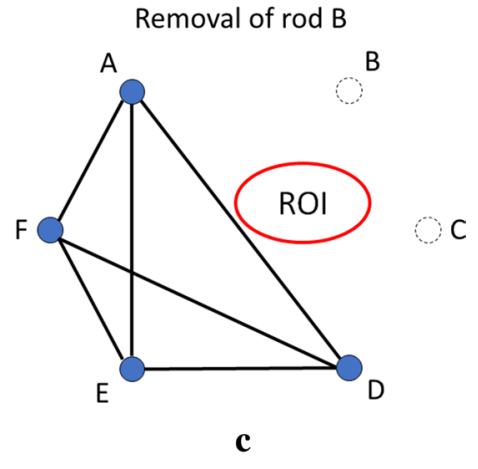

c

Fig. 11 Schematic representation of rod coupling in the presence of 6 rods and the effect of rod removal on the metal artefacts. a Complete graph. b Resulting coupling after removal of rod C. $\mathbf{c}$ Resulting coupling after the removal of rods B and C

broader and closer together, the ability to resolve features relating to those peaks diminishes. It can be seen that for Figs. 8 and 9, the data in Tables 3 and 4 demonstrate that the application of MARS reduces the level of artefacts but also affects the separability between the peaks.

From our perspective, the key point of this study is an appreciation of the phenomenon of rod coupling. Before CT scanning a limb with a circular external fixator, a thoughtful analysis should be made as to the selection of which rods to remove, or if they cannot be removed, how to configure them to avoid the worst effect of the metal artefacts on the region of interest. In our opinion, the best option is to temporarily reduce the number of rods to the bare minimum and/ or to avoid rods at opposite sides of the ROI. That way, the surgeon can optimise image quality in the ROI, making it potentially easier to take medical decisions that impact the treatment and/or recovery of the patient.

\section{Removal of rods}

A rod-coupling line goes straight from every rod to every other rod (Fig. 11a; case with six rods). When a rod is removed, all lines connected to it are also deleted. One wishes to remove as many rods as are needed, to not have any rod-coupling lines going through the ROI. For example, if the maximum number of rods that can be left remaining is 3 , then one could remove $\mathrm{B}+\mathrm{D}+\mathrm{F}$, so as to have no rod couplings going through the ROI. A systematic process would be as follows:

1. Identify the rod(s) with the greatest number of lines passing through the ROI. In Fig. 11, B and C are the only rods with more than one line passing through the ROI. So, rod C is removed (Fig. 11b).

2. Again, we identify the $\operatorname{rod}(\mathrm{s})$ with the greatest number of lines passing through the ROI. Removing rod B will 
reduce the number of rod couplings in the ROI by two (Fig. 11c). If more than one rod reduces the number of rod couplings in the ROI by the same amount, a different criterion can be chosen, for example, if a more rigid frame can be obtained by removing one and not the other.

\section{Compliance with ethical standards}

Conflict of interest The authors declare no conflict of interest, and no funds have been received in connection with this study.

Ethical approval This experiment used a sheep leg that had been used in experiments conducted with approval from the Monash University (Australia) Animal Ethics Committees. All experiments were performed in accordance with relevant guidelines and regulations. The sheep was humanely killed in line with approved guidelines and the carcasses scavenged for this experiment.

Informed consent For this type of study (animal research) formal patient consent is not required.

Open Access This article is distributed under the terms of the Creative Commons Attribution 4.0 International License (http://creativeco mmons.org/licenses/by/4.0/), which permits unrestricted use, distribution, and reproduction in any medium, provided you give appropriate credit to the original author(s) and the source, provide a link to the Creative Commons license, and indicate if changes were made.

\section{References}

1. Fragomen AT, Rozbruch SR (2007) The mechanics of external fixation. HSS J 3(1):13-29

2. Wright J, Sabah SA, Patel S, Spence G (2017) The silhouette technique: improving post-operative radiographs for planning of correction with a hexapod external fixator. Strategies Trauma Limb Reconstr 12(2):127-131

3. Coupal TM, Mallinson PI, McLaughlin P, Nicolaou S, Munk PL, Ouellette H (2014) Peering through the glare: using dual-energy
CT to overcome the problem of metal artefacts in bone radiology. Skelet Radiol 43(5):567-575

4. Huang JY, Kerns JR, Nute JL, Liu X, Balter PA, Stingo FC, Followill DS, Mirkovic D, Howell RM, Stingo FC, Kry SF (2015) An evaluation of three commercially available metal artifact reduction methods for CT imaging. Phys Med Biol 60(3):1047

5. Kotsenas AL, Michalak GJ, DeLone DR, Diehn FE, Grant K, Halaweish AF, Krauss A, Raupach R, Schmidt B, McCollough $\mathrm{CH}$, Fletcher JG (2015) CT metal artifact reduction in the spine: can an iterative reconstruction technique improve visualization? Am J Neuroradiol 36(11):2184-2190

6. Morsbach F, Bickelhaupt S, Wanner GA, Krauss A, Schmidt B, Alkadhi H (2013) Reduction of metal artifacts from hip prostheses on CT images of the pelvis: value of iterative reconstructions. Radiology 268(1):237-244

7. Hsieh J (2009) Computed tomography: principles, design, artifacts, and recent advances. Bellingham, Washington

8. Buckwalter KA, Lin C, Ford JM (2011) Managing postoperative artifacts on computed tomography and magnetic resonance imaging. Semin Musculoskelet Radiol 15(4):309-319

9. Gupta A, Subhas N, Primak AN, Nittka M, Liu K (2015) Metal artifact reduction: standard and advanced magnetic resonance and computed tomography techniques. Radiol Clin 53(3):531-547

10. Meyer E, Raupach R, Lell M, Schmidt B, Kachelrieß M (2012) Frequency split metal artifact reduction (FSMAR) in computed tomography. Med Phys 39(4):1904-1916

11. Berg BV, Malghem J, Maldague B, Lecouvet F (2006) Multidetector CT imaging in the postoperative orthopedic patient with metal hardware. Eur J Radiol 60(3):470-479

12. Lee YH, Park KK, Song HT, Kim S, Suh JS (2012) Metal artefact reduction in gemstone spectral imaging dual-energy CT with and without metal artefact reduction software. Eur Radiol 22(6): 1331-1340

13. Pessis E, Campagna R, Sverzut JM, Bach F, Rodallec M, Guerini H, Feydy A, Drapé JL (2013) Virtual monochromatic spectral imaging with fast kilovoltage switching: reduction of metal artifacts at CT. Radiogr 33(2):573-583

14. Gao F, Tao D, Gao X, Li X (2015) Learning to rank for blind image quality assessment. IEEE Trans Neural Netw Learn Syst 26(10):2275-2290

15. Hou W, Gao X, Tao D, Li X (2015) Blind image quality assessment via deep learning. IEEE Trans Neural Netw Learn Syst 26(6):1275-1286

16. Xue W, Mou X, Zhang L, Bovik AC, Feng X (2014) Blind image quality assessment using joint statistics of gradient magnitude and Laplacian features. IEEE Trans Image Process 23(11):4850-4862 